\title{
Aprendizaje basado en proyectos en la asignatura Energía y Desarrollo Sostenible
}

\begin{abstract}
Isabel Martóna ${ }^{a}$, José Felipe Villanueva ${ }^{b}$, Sergio Gallardo ${ }^{c}$, Sofia Carlos Alberola ${ }^{d}$, Ana Sánchez ${ }^{a}$ Dpto. de Estadística e Investigación Operativa Aplicadas y Calidad, Universitat Politècnica de València, Spain, email: ismarllu@upvnet.upv.es; ${ }^{b}$ Dpto. de Ingeniería Química y Nuclear, Universitat Politècnica de València, Spain, email:jovillo0@upvnet.upv.es; 'Dpto. de Ingeniería Química y Nuclear, Universitat Politècnica de València, Spain, email: sergalbe@iqn.upv.es; ${ }^{\mathrm{d}}$ Dpto. de Ingeniería Química y Nuclear, Universitat Politècnica de València, Spain, email: scarlos@iqn.upv.es; ${ }^{e}$ Dpto. de Estadística e Investigación Operativa Aplicadas y Calidad, Universitat Politècnica de València, Spain, email: aisanche@eio.upv.es
\end{abstract}

\begin{abstract}
In the standards and guidelines for quality assurance in the European Higher Education Area (EHEA), is established that one of the keys to combining the knowledge and skills of the learners is the implementation of new pedagogical active methodologies that are more student-centered, and learning based on competences. One of the most widely used and effective active learning methodologies is Project-Based Learning (PBL). The PBL is a practical pedagogical methodology, in which the student should develop a project focused on the investigation of solving a real problem by applying the theoretical concepts from a practical point of view.
\end{abstract}

In this work, projects implanted using the PBL methodology are aligned with the Agenda 2030. This Agenda for Sustainable Development, approved in 2015, establishes that the universities must play a major role in compliance with this, in due consideration of their responsibility in relation to training, research, relations with society and a model for higher education governance. In this agenda, 17 Sustainable Development Goals (SDGs).. This proposal is focused on Goal 7 "Affordable and clean energy".

In the frame of the PBL methodology and integrating the SDGs of the Agenda 2030, the main goal of this work is the development of project-based learning in the subject "Energy and Sustainable Development" of the bachelor's degree in Energy Engineering in the Universitat Politècnica de València. Thus, this paper presents the developed methodology, the achieved results and the initial conclusions obtained.

Keywords: Project Based Learning (PBL), Energy, Engineering, Sustainable development, Statistic

\section{Resumen}

En las normas y directrices para el aseguramiento de la calidad en el Espacio Europeo de Educación Superior (EEES), se establece que una de las claves para combinar el conocimiento y las habilidades de los alumnos es la implementación de nuevas metodologías pedagógicas activas que estén más centradas en el alumno, y aprendizaje basado en competencias. Una de las metodologías de aprendizaje activo más utilizadas y eficaces es el aprendizaje basado en proyectos (PBL). El PBL es una metodología pedagógica práctica, en la que el alumno debe desarrollar un proyecto centrado en la investigación para resolver un problema real mediante la aplicación de los conceptos teóricos desde un punto de vista práctico. 
En este trabajo, los proyectos implantados utilizando la metodología PBL están alineados con la Agenda 2030. Esta Agenda para el Desarrollo Sostenible, aprobada en 2015, establece que las universidades deben desempeñar un papel importante en el cumplimiento de esto, teniendo debidamente en cuenta su responsabilidad en relación con formación, investigación, relaciones con la sociedad y un modelo para la gobernanza de la educación superior. En esta agenda, se propusieron 17 Objetivos de Desarrollo Sostenible (ODS). Esta propuesta se centra en el Objetivo 7 "Energía limpia y asequible".

En el marco de la metodología PBL e integrando los ODS de la Agenda 2030, el objetivo principal de este trabajo es el desarrollo del aprendizaje basado en proyectos en la asignatura "Energía y Desarrollo Sostenible" del Grado de Ingeniería de la Energía en la Universitat Politècnica de València. Por lo tanto, este artículo presenta la metodología desarrollada, los resultados obtenidos y las conclusiones iniciales obtenidas.

Palabras clave: Aprendizaje Basado en Proyectos (ABP), Energía, Desarrollo sostenible, Ingeniería, Estadística

\section{Introducción}

En las normas y directrices para el aseguramiento de la calidad en el Espacio Europeo de Educación Superior (EEES), se establece que una de las claves para combinar el conocimiento y las habilidades de los alumnos es la implementación de nuevas metodologías pedagógicas activas que estén más centradas en el alumno, y aprendizaje basado en competencias (Garcia, 2017). Una de las metodologías de aprendizaje activo más utilizadas y eficaces es el aprendizaje basado en proyectos (PBL). El PBL es una metodología pedagógica práctica, en la que el alumno debe desarrollar un proyecto centrado en la investigación para resolver un problema real mediante la aplicación de los conceptos teóricos desde un punto de vista práctico (Huff, 2016).

Entre las características de esta metodología se pueden resumir las siguientes: es una metodología centrada en el alumno y en su aprendizaje, parte de un planteamiento de un caso real. Además, el ABP, favorece el compromiso del alumno con su aprendizaje y con el de su grupo de trabajo. Un aspecto que resulta fundamental es que estén implicadas distintas área para potenciar el carácter interdisciplinar (Cuñas, 2016).

En el caso del Grado de Ingeniero de la Energía (GIE), de la Univesitat Politècnica de València (UPV), los autores del presente trabajo han detectado en diversas asignaturas carencias en conceptos básicos que dificultan el óptimo desarrollo de ciertas competencias específicas. En este marco, el departamento de Ingeniería Química y Nuclear (DIQN) y el departamento de Estadística e Investigación Operativa Aplicadas y Calidad (DEIOAC) han trabajado conjuntamente para identificar debilidades, amenazas, fortalezas y oportunidades en algunas asignaturas de GIE y se ha podido constatar que en la asignatura de primer curso "Estadística" y en la asignatura de segundo curso "Energía y Desarrollo Sostenible" existe una clara oportunidad de mejora en el desempeño docente. Para ello, se proponer optimizar los recursos didácticos fomentando las sinergias entre las dos asignaturas. Más concretamente, el problema se ha puesto de manifiesto al tratar de aplicar técnicas estadísticas a problemas relacionados con la generación de energía. En trabajos anteriores de los autores (Marton, 2019) se muestran las actividades desarrolladas para la asignatura de primer curso y los resultados obtenidos mediante esta metodología. Se desarrollaron actividades relacionadas el tema de producción de energía eólica,utilizando la distribución estadística que caracteriza el comportamiento de la velocidad del viento en frecuencia, función de distribución Weibull. Por otra parte, también se ha constatado en los últimos años y en trabajos previos de los autores, que el 
alumnado se siente especialmente motivado en temas de energías renovables (como la eólica). Aunando estos dos hechos, se han podido identificar una debilidad (carencias de base teórica y técnica) y una fortaleza (atracción por las energías renovables) en las asignaturas mencionadas anteriormente.

Además, es importante que desde el punto de vista de los trabajos se tenga en cuenta el contexto de la Agenda de Desarrollo 2030 y los Objetivos de desarrollo Sostenible (ODS) (Naciones Unidas, 2019). En el año 2015, en el marco de Naciones Unidas, se acordó de manera unánime la llamada Agenda de Desarrollo 2030 donde se persigue el vínculo del desarrollo sostenible con procesos económicos, sociales y ambientales mediante 17 Objetivos de Desarrollo Sostenible (ODS) que representan un plan a favor de las personas, del planeta y de la prosperidad, aspirando a que para el año 2030 hayamos situado al mundo y a sus sociedades en un camino hacia un futuro mejor (Fig. 1).
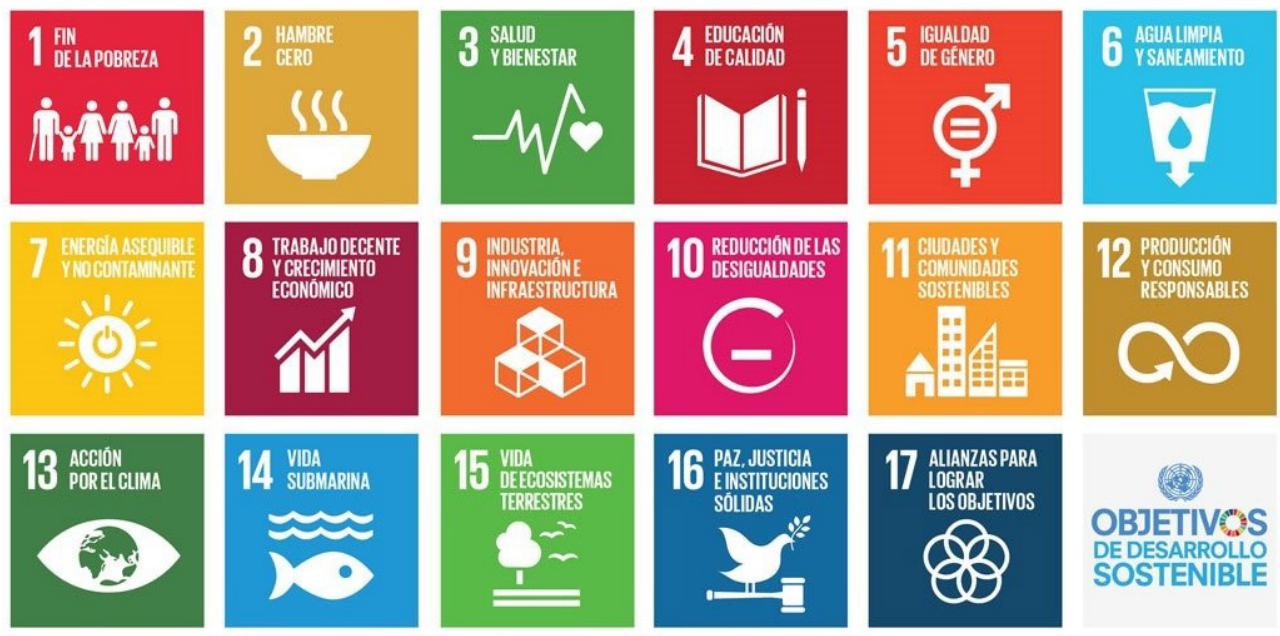

OBJETIVOS

DEDESARROLLO SOSTENIBLE

Fig. 1. Objetivos de desarrollo sostenible.

Estos ODS sitúan a la educación de calidad como pieza clave para el desarrollo sostenible a través del ODS 4. Alguna de las metas propuestas para este ODS involucra directamente a las universidades. Por ejemplo mediante la meta 4.7, en la cual se considera que las universidades juegan un papel fundamental ya que proporcionan las soluciones, el conocimiento y las ideas innovadoras a los ODS, son los encargadas de formar a los actuales y futuros ejecutores y responsables de implementar los ODS, sirven de modelo sobre cómo apoyar, adoptar e implementar los ODS en la gobernanza, las políticas de gestión y la cultura universitaria y desarrollan liderazgos intersectoriales que orientan a los ODS.

Además, en el contexto del grado en el que se desarrolla esta innovación, es importante la alineación de los trabajos de ambas asignaturas con el ODS 7 "Energía limpia y asequible". Estas asignaturas podrían utilizarse como una herramienta importante para el desarrollo del ODS 7 en la toma de decisiones a la hora de diseñar y planificar sistemas de energía limpia y que favorezcan un desarrollo sostenible, por ejemplo, en toma de decisiones a la hora de diseñar y planificar sistemas de energía limpia y que favorezcan un desarrollo sostenible.

En el marco de la metodología del PBL e integrando los ODS de la Agenda 2030, el objetivo principal de este trabajo es el desarrollo del aprendizaje basado en proyectos en la asignatura "Energía y Desarrollo Sostenible" de la licenciatura en Ingeniería Energética en la Universitat Politècnica de València. Por lo tanto, este artículo presenta la metodología desarrollada, los resultados obtenidos y las conclusiones iniciales obtenidas. 


\section{Objetivos}

El objetivo general del trabajo es el de diseñar actividades enfocadas al aprendizaje basado en proyectos y, desarrollar y establecer metodologías, definir técnicas de evaluación apropiadas de evaluación centradas en el aprendizaje basado en proyectos en la asignatura de "Energía y Desarrollo Sostenible", potenciando el uso de los resultados de aprendizaje de la asignatura de "Estadística" en el Grado de Ingeniero de la Energía.

Este trabajo se enmarca en un Proyecto de Innovación y Mejora Educativa de la Universitat Politècnica de València de dos años de duración (2018-2020) aplicado al Grado de Ingeniero de la Energía (GIE). Actualmente, el proyecto está en su segundo año de ejecución, en el presente trabajo se van a mostrar las actividades, metodología y los resultados obtenidos durante este periodo. Además, se muestran de forma preliminar algunos resultados obtenidos de implementación de dichas actividades.

Los objetivos específicos del trabajo son los siguientes:

- Comprobar si las actividades desarrolladas en la asignatura de "Estadística" han sido efectivas para alcanzar los conocimientos básicos necesarios.

- Diseñar actividades específicas en la asignatura de "Energía y Desarrollo Sostenible" de forma que se integre de forma transversal con los objetivos de aprendizaje de la asignatura alcanzados previamente en la asignatura de "Estadística".

- Alinear las actividades en el ODS 7 las cuales fomenten la utilización de fuentes de energía renovable.

- Diseñar actividades que permitan al alumno la adquisición, tanto de las competencias específicas como de las transversales de la asignatura "Energía y Desarrollo Sostenible".

En los siguientes apartados se presenta el contexto y el desarrollado de la innovación, la metodología y los primeros resultados obtenidos. Por último, se incluyen las conclusiones del estudio.

\section{Desarrollo de la innovación}

Siguiendo con la experiencia iniciada en el curso 2018-2019, en la segunda anualidad del proyecto PIME en el cual se encuentra enmarcado el presente trabajo, se aplica la metodología de Aprendizaje Basado en Proyectos a la asignatura de segundo curso de GIE (Energía y Desarrollo Sostenible, EyDS).

En los últimos años, se ha detectado que los alumnos presentan problemas a la hora de aplicar los conceptos teóricos vistos en el primer curso en la asignatura de Estadística con la aplicabilidad práctica de problemas reales presentados en la asignatura de $2^{\circ}$ curso. Este problema implica perder oportunidades de sinergia entre las distintas asignaturas para trabajar de forma más efectiva algunas competencias. En este contexto, el objetivo principal de este proyecto es el de desarrollar un proyecto que integre las competencias específicas y transversales de estas asignaturas para que el alumno pueda aplicar de forma práctica los conceptos en un contexto real. El alumno al mismo tiempo que utiliza las herramientas estadísticas desarrolla dos capacidades: la de calcular y la de trabajar en un proyecto del sector energético.

La experiencia que se propone en la asignatura de EyDS es el diseño de un parque eólico terrestre mediante una sesión de práctica de informática. Los objetivos de la práctica son los siguientes:

- Diseñar de manera preliminar un parque eólico y estimar la energía que puede producir

- Determinar la viabilidad económica del proyecto. 
La práctica, con una duración de tres horas, comienza con la presentación de los objetivos anteriores y con una breve descripción por parte del profesor del panorama energético de la Comunitat Valenciana en términos de producción de energía de origen renovable. A continuación, se describe brevemente el Plan Eólico de la Comunitat Valenciana, describiendo la compartimentación territorial que se detalla en el propio plan. En este punto de la exposición, se enumeran los principales parques eólicos, su capacidad instalada, año de construcción y promotor. Con todo ello se pretende que el alumnado tenga una visión lo más global posible de la situación actual del sector eólico en nuestra comunidad. Finalizada esta fase introductoria, se establece un turno abierto de participación para determinar con la técnica de brainstorming cuáles son los requisitos mínimos que hay que cumplir para elegir la ubicación de un parque eólico. Esta fase de la práctica es muy interesante porque el alumnado es capaz de identificar la mayoría de los factores a tener en cuenta. El profesor se limita a clasificar los factores identificados en tres grandes categorías:

- Requisitos administrativos.

- Requisitos técnicos.

- Requisitos medioambientales.

Una vez identificados los factores a tener en cuenta, se plantea el problema a resolver en la práctica. Se trata de diseñar un parque eólico de $16 \mathrm{MW}$ en una zona apta para su ubicación dentro de la Comunitat Valenciana. Se ha elegido la zona eólica 4 del Plan Eólico de la Comunitat Valenciana (que incluye los municipios de Albocàsser, Tirig, Catí, Xert, etc).

Se propone considerar un modelo de aerogeneradores de una determinada marca comercial. Se facilita el catálogo comercial de dicho aerogenerador con las especificaciones técnicas que necesitan para resolver la práctica: curva de potencia, potencia nominal, diámetro del rotor, altura del buje y pérdidas globales. En la identificación de los requisitos técnicos, se determinó que la separación entre aerogeneradores es un parámetro esencial para reducir las pérdidas por afección entre turbinas. Una forma de limitar dichas pérdidas al $5 \%$ es establecer una distancia mínima intrafila de 2 diámetros de rotor entre aerogeneradores. Teniendo en cuenta la potencia nominal de cada máquina y la potencia nominal del parque, los alumnos determinan el número de turbinas y, por tanto, la longitud mínima del parque, suponiendo que todas las máquinas se ubican en la misma fila. Este parámetro es esencial en el desarrollo de la práctica porque establece la longitud mínima de la colina que deben buscar para las posibles ubicaciones del parque. Por otro lado, se les remarca la importancia de que la colina tenga una buena orientación respecto de la dirección predominante del viento.

Con estas dos ideas: longitud de colina y dirección predominante del viento (según la Rosa de Vientos del lugar), da comienzo la segunda parte de la práctica. Esta parte se realiza de forma completamente autónoma por parte del grupo. En este punto, se proporciona a los alumnos las herramientas necesarias para proponer posibles ubicaciones del parque: el mapa cartográfico de la Comunitat Valenciana (versión de visor on-line) y el atlas eólico (Global Wind Atlas). Para delimitar geográficamente las posibles soluciones, se reduce zona de estudio al límite municipal entre Catí y Albocasser. Para ello se utiliza el visor del Instituto Cartográfico Valenciano https://visor.gva.es/visor/ ó el Visor Map Windows 5.

Los alumnos deben buscar colinas que cumplen con los requisitos de orientación, longitud y altitud determinadas anteriormente. Esta parte de la práctica resulta muy interesante porque implica una búsqueda que no tiene una única solución. Los alumnos experimentan que el proceso de búsqueda implica un proceso de cribado, pero también un proceso de prueba y error, porque no se puede garantizar a partir del mapa cartográfico, que una colina de dimensiones adecuadas cumpla con el criterio de orientación óptima respecto de la dirección predominante del viento hasta que no se verifica con el Atlas Eólico. 
A todas estas restricciones, se deben añadir las restricciones medioambientales. Se hace hincapié que no se pueden proponer ubicaciones que estén dentro de una figura ambiental: Red Natura 2000 y Espacios Naturales Protegidos (Fig. 2).

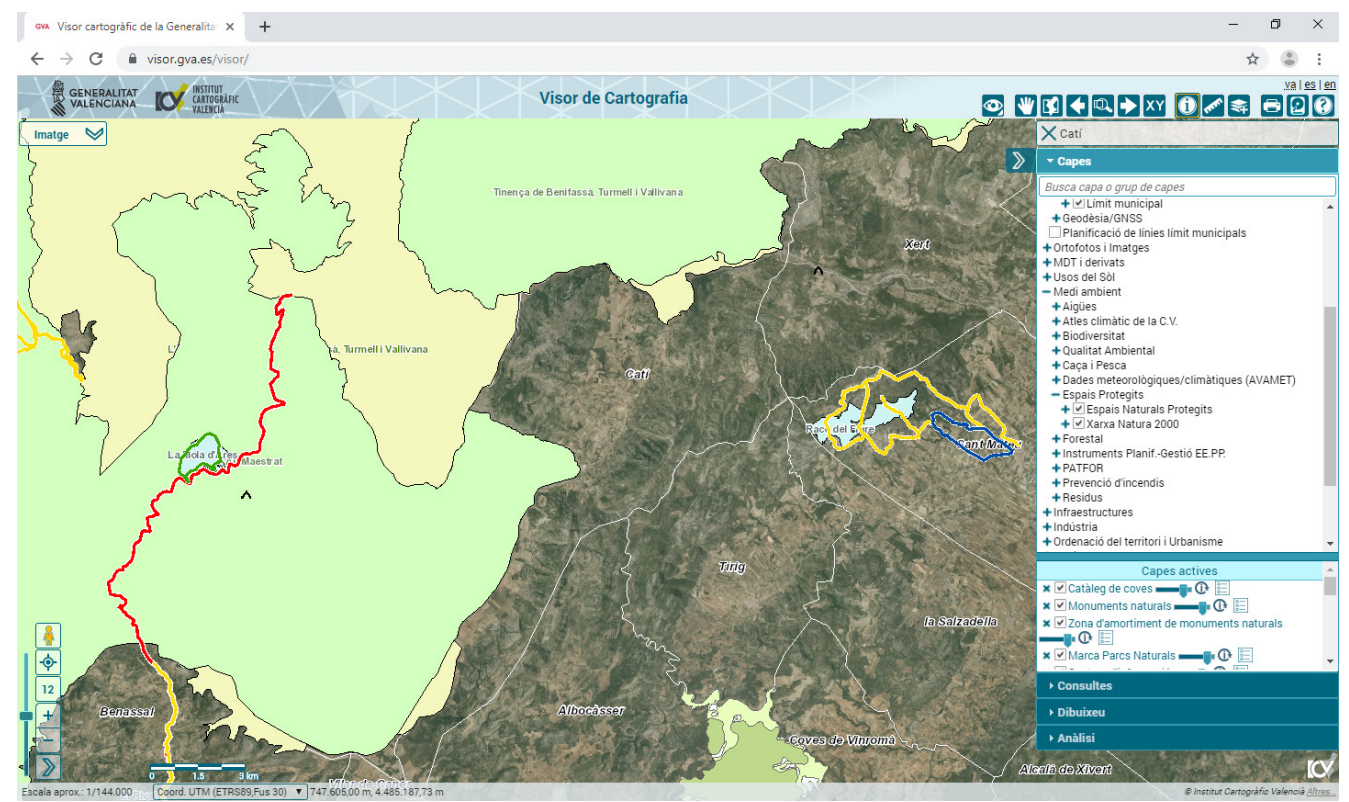

Fig. 2. Espacios protegidos en la zona de interés. Visor del mapa cartográfico valenciano.

Se establece un tiempo máximo de 15 minutos para localizar una colina que cumpla con todos los criterios predefinidos. En caso de que algún alumno no logre encontrar una colina adecuada, se le indica una para que continúe la práctica (Fig. 3). Se comprueban las condiciones eólicas de la colina con la herramienta Global Wind Atlas (Fig. 4).

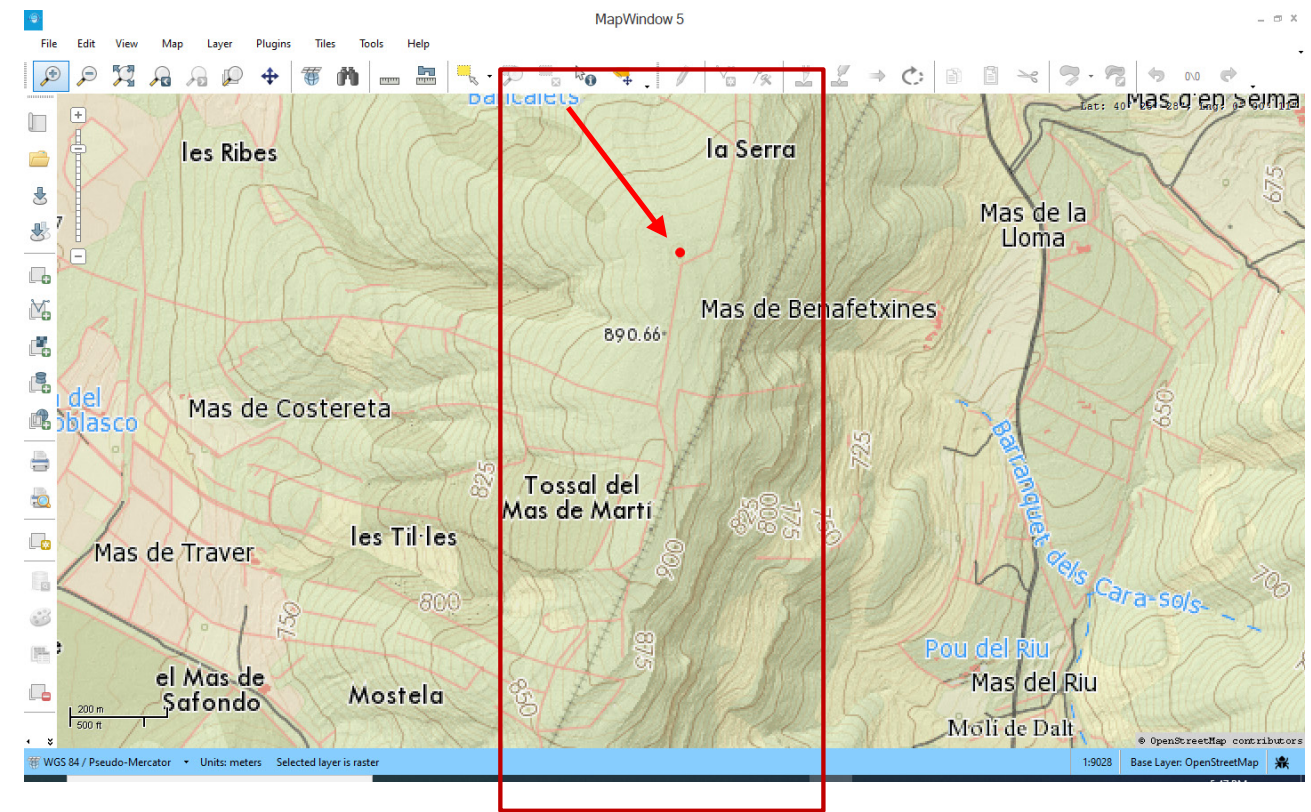

Fig. 3. Colina propuesta. Visor del mapa cartográfico valenciano. 


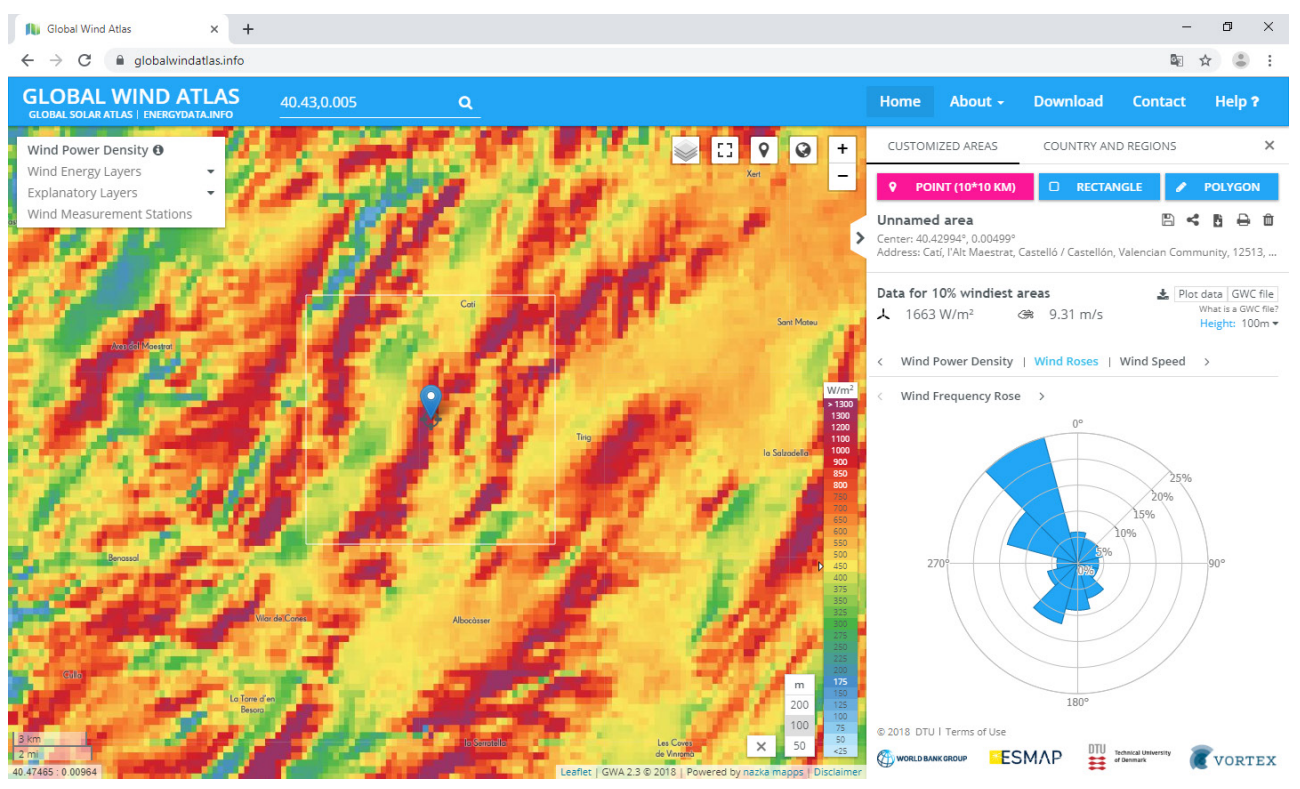

Fig. 4. Densidad de potencia de la colina propuesta. Global Wind Atlas.

Una vez escogida la colina, se consultan los parámetros eólicos de interés con Global Wind Atlas: velocidad media del viento, densidad de potencia, rosa de vientos en frecuencias y parámetros A y K de la distribución de Weibull.

Es en este punto donde se necesitan los conocimientos adquiridos en Estadística de primer curso. Los alumnos deben relacionar el concepto de distribución Weibull con lo aprendido en Estadística. Fruto de la coordinación vertical entre ambas asignaturas, se consigue un ahorro de tiempo ya que no es necesario reincidir en este concepto. Además, en Estadística, los alumnos han estudiado el significado y utilidad de este tipo de distribución con ejemplos que han proporcionado los profesores que imparten docencia en la asignatura de segundo curso.

Una vez conseguida toda esta información, se procede al cálculo de la energía producida por el parque, realizando la convolución de la curva de potencia, la distribución Weibull para el lugar de interés y el número de turbinas.

Con objeto de motivar más al alumnado, se hace un ranking con la energía calculada por cada estudiante, obteniendo entre todo el grupo de prácticas una clasificación geográfica en función de la colina escogida y de la energía bruta producida.

En la última parte de la práctica se determina si el parque es viable económicamente. Para ello se calcularán los parámetros VAN y TIR utilizando el programa RETSCREEN, haciendo uso de la información obtenida en los apartados anteriores (Fig. 5).

Teniendo en cuenta todos los cálculos realizados en cada parte de la práctica, los alumnos adquieren la competencia de evaluar económica el parque. En este punto y casi para finalizar la práctica, se hace una puesta en común de la rentabilidad que obtenido cada uno. Resulta muy llamativo para los alumnos comprobar que únicamente una mala decisión de ubicación del parque puede causar que el proyecto no sea económicamente viable. 
La experiencia se ha realizado por primera vez en el curso 2019-2020 y ha resultado muy positiva tanto en la respuesta de los alumnos de cara a afrontar el proyecto, como en la motivación adicional que representa presentar un proyecto de mayor rentabilidad que otros compañeros.

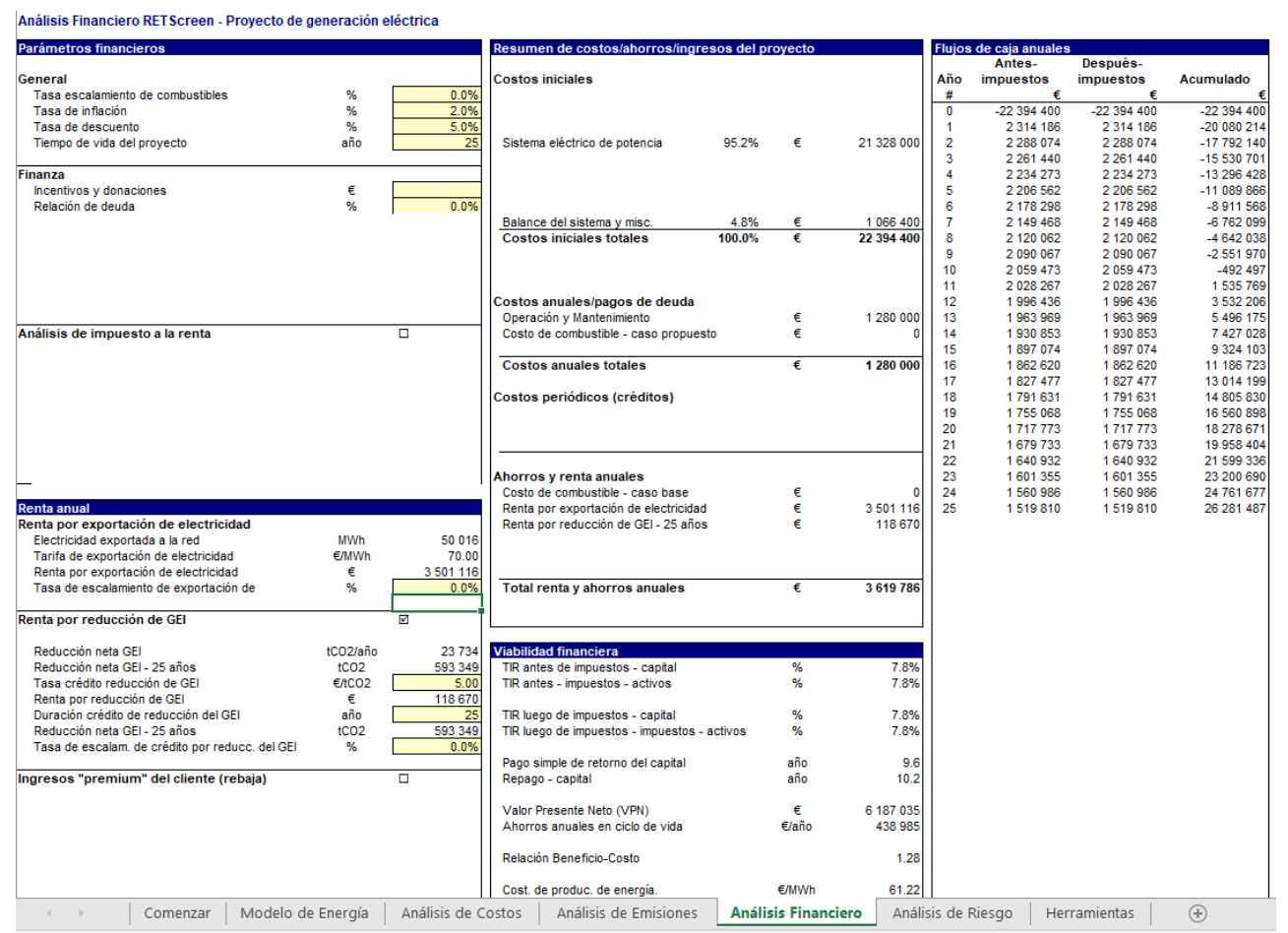

Fig. 5. Ejemplo de pantalla de cálculo de viabilidad económica. Retscreen (C)

En este año de puesta en marcha de la práctica que utiliza la metodología de Aprendizaje basado en proyectos, la evaluación se ha realizado mediante la elaboración de una pequeña memoria ( 5 páginas aproximadamente) explicando las distintas fases de elaboración del proyecto.

Sin embargo, conscientes del tiempo limitado del que disponen los alumnos para elaborar la memoria de prácticas, para el próximo curso se propondrá contestar un cuestionario vía herramientas "Exámenes" de Politformat (UPV) con preguntas cortas de libre formato y preguntas de respuesta numérica, de forma que al imprimir el cuestionario, los alumnos dispondrán de un procedimiento para determinar la viabilidad económica de un parque, particularizado a la ubicación elegida por cada uno.

\section{Resultados}

Una vez llevada a cabo la actividad dentro de la asignatura se ha procedido ha realizar un estudio preliminar de los resultados obtenidos. Para ello, en previsión de la realización de la actividad, el año anterior a los alumnos de la a signatura de "Energía y Desarrollo Sostenible" se les sometió a un cuestionario realizado sobre un plataforma online en horario lectivo, donde para cada uan de las preguntas se les daban cuatro opciones a elegir, existiendo solo una pregunta correcta posible, y con un tiempo límite por pregunta. Ese mismo cuestionario se ha pasado a los alumnos de este curso tras la realización de la experiencia de aprendizaje basado en proyectos. 
El cuestionario constaba de 20 preguntas englobadas bajo tres dimensiones donde se indagaba sobre conceptos puramente teóricos, de cálculo y de toma de decisiones bajo premisas.

El momento de evaluación no se había avisado en ambo casos con anterioridad, aunque sí que eran conocedores que se produciría tras la realización de la actividad.

La participación fue elevada en ambos casos: 31 y 36 alumnos.

Se mantuvieron el resto de variables de contorno estables de un año a otro: mismo temario, mismo profesroado, mismas actividades en clase, mismos horarios, mismo instante de evaluación. La única actividad diferente fue la presentada en este trabajo, bajo el desarrollo conceptual y práctico como proyecto, y la actividad que el año anterior habían realizado los mismos alumnos en la asignatura de "Estadística" de $1^{\circ}$ de GIE (Tabla 1).

Tabla 1. Ficha cuestionario

\begin{tabular}{|c|c|}
\hline $\mathrm{N}^{\circ}$ preguntas & 20 \\
\hline Dimensiones & 3 \\
\hline $\begin{array}{l}\mathrm{N}^{\mathrm{o}} \text { opciones por } \\
\text { pregunta }\end{array}$ & 4 \\
\hline $\mathrm{N}^{\mathrm{o}}$ alumnos & $31-36$ \\
\hline Control de tiempo & Sí \\
\hline Resto Variables & Constantes \\
\hline
\end{tabular}

Reseñar en primer lugar que todos los participantes respondieron a todas las preguntas. Respecto a las contrestaciones, a nivel general de los resultados obtenidos se ha apreciado un incremento porcentual de un $40.85 \%$ en el porcertanje de aciertos en todas las preguntas. Esta diferencia resulta claramente significativa al plantear la hipótesis sobre si ha habido un aumento o no en el porcentaje de aciertos con valores de p-valor en la práctica nulos.

Respecto a cada una de las preguntas formuladas (Tabla 2) se ha observado que ha habido incrementos significativos en los porcentajes de aciertos en 8 de las 20 preguntas, con p-valores, en la hipóstesis de incremento en dicho porcentaje, entre el 0.02 y 0.0011 . De las 12 preguntas restantes 2 de ellas presnta un incremento de $46 \%$ y el $100 \%$, incremento que las pone en el borde de los límites de aceptabilidad de hipótesis con p-valoes cercanos al 0.05 .

Tabla 2. Resultados del análisis por preguntas y dimensiones

\begin{tabular}{cccc}
\hline & Incremento & Incremento porcentual & p-valor \\
\hline Q1 & 14.96 & 46.37 & 0.0840 \\
Q2 & 6.99 & 72.21 & 0.2182 \\
Q3 & 38.17 & 69.60 & 0.0053 \\
Q7 & 2.42 & 10.72 & 0.4199 \\
Q9 & 27.15 & 325.93 & 0.0103 \\
Q17 & 8.69 & 62.56 & 0.2052 \\
Dimensión Cálculo & 16.40 & 95.14 & 0.0474 \\
\hline
\end{tabular}


Aprendizaje basado en proyectos en la asignatura Energía y Desarrollo Sostenible

\begin{tabular}{cccc}
\hline Q4 & 2.42 & 10.72 & 0.4199 \\
Q5 & 7.17 & 14.82 & 0.3428 \\
Q8 & 21.33 & 330.70 & 0.0198 \\
Q11 & -4.92 & -13.87 & 0.6370 \\
Q12 & 24.64 & 109.12 & 0.0465 \\
Q13 & 35.66 & 1104.02 & 0.0011 \\
Q14 & -5.28 & -9.09 & 0.6141 \\
Dimensión Teórica & 11.57 & 41.17 & 0.2101 \\
\hline Q6 & -0.72 & -1.59 & 0.5175 \\
Q10 & 41.76 & 215.81 & 0.0042 \\
Q15 & 19.98 & 123.87 & 0.0578 \\
Q16 & -11.74 & -18.20 & 0.7350 \\
Q18 & 0.09 & 0.47 & 0.4967 \\
Q19 & 56.53 & 194.73 & 0.0014 \\
Q20 & 35.22 & 546.05 & 0.0022 \\
Dimensión Crítica & 20.16 & 70.56 & 0.0475 \\
\hline
\end{tabular}

Respecto a cada dimensión (teórica, práctica, toma de decisiones o capacidad crítica), aunque en casi todas elas preguntas ha habido un incremento en perocentaje, en todas las dimensiones se han presentado entre dos o tres preguntas que incrementaban significativamente el porcentaje de aciertos, en especial en la dimensión de cálculo donde se mejoran 3 de las 7 preguntas (Figuras 6, 7 y 8, Tabla 2).

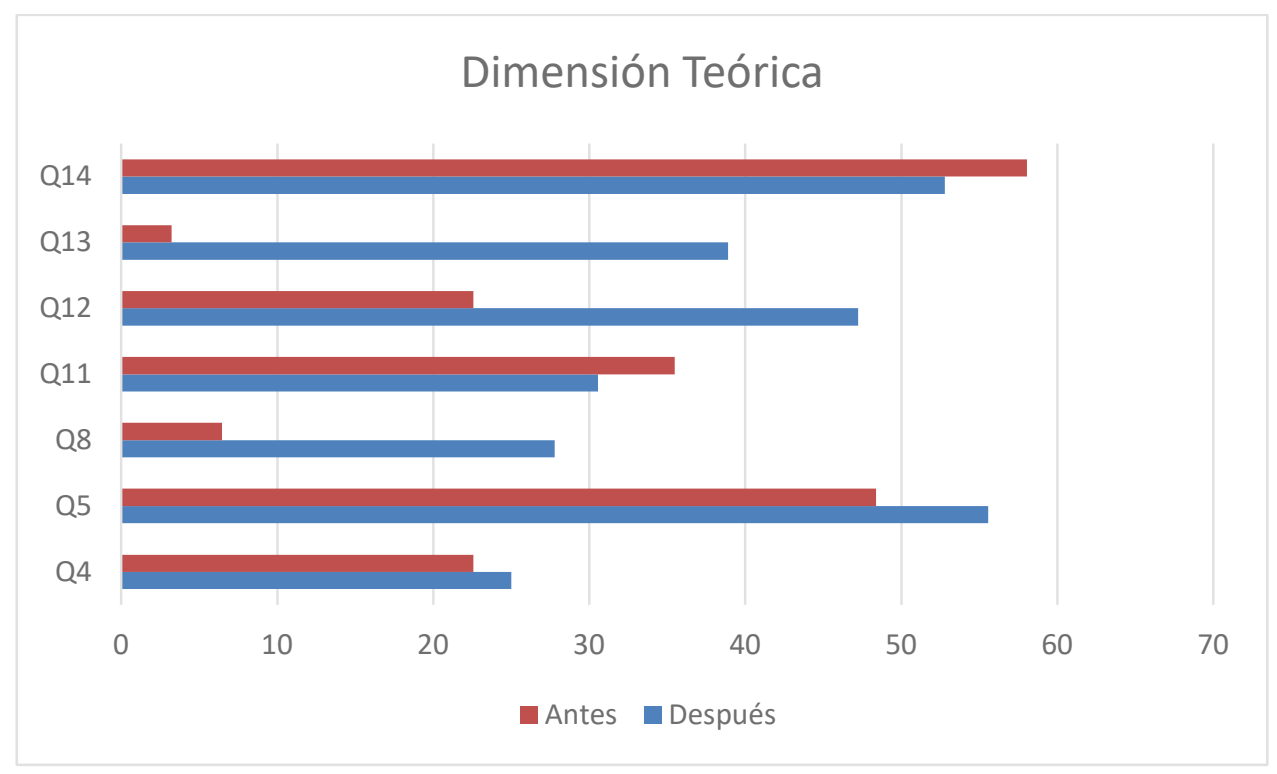

Fig. 6. Porcentajes aciertos a las preguntas dentro de la dimensión teórica 


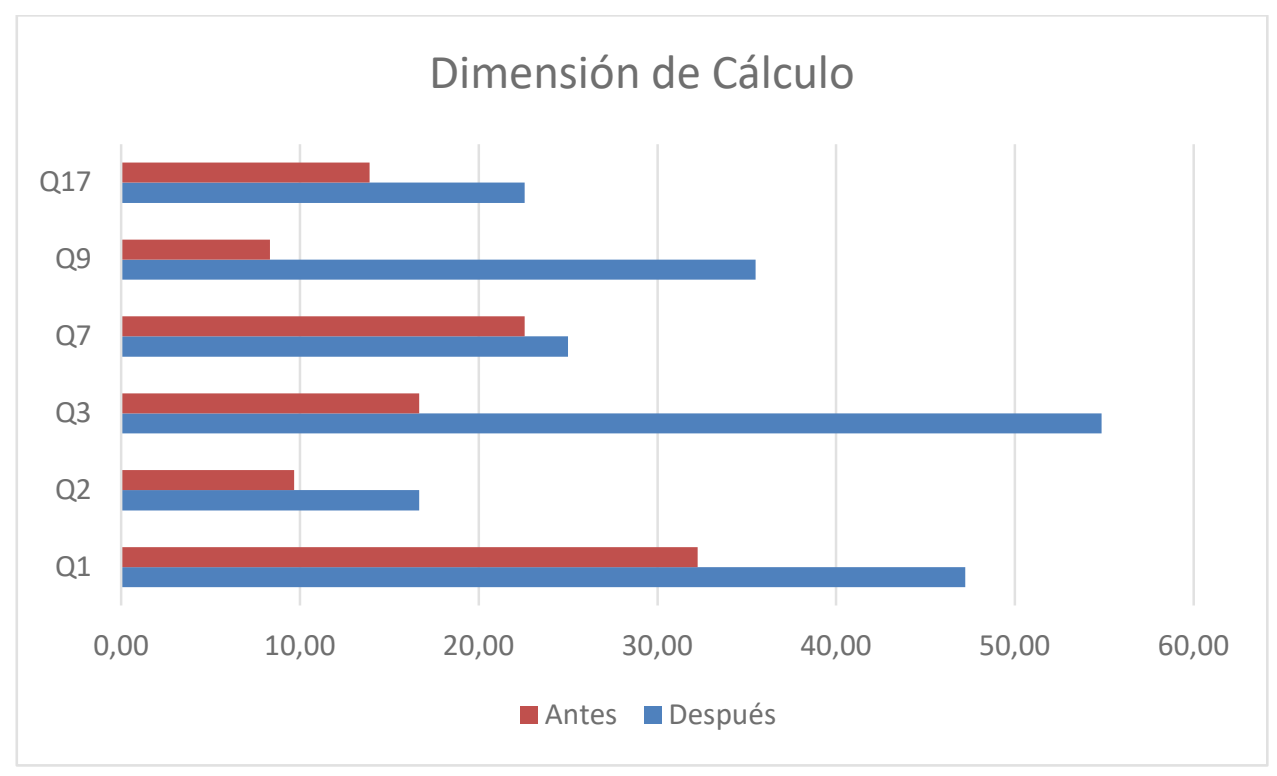

Fig. 7. Porcentajes aciertos a las preguntas dentro de la dimensión de cálculo

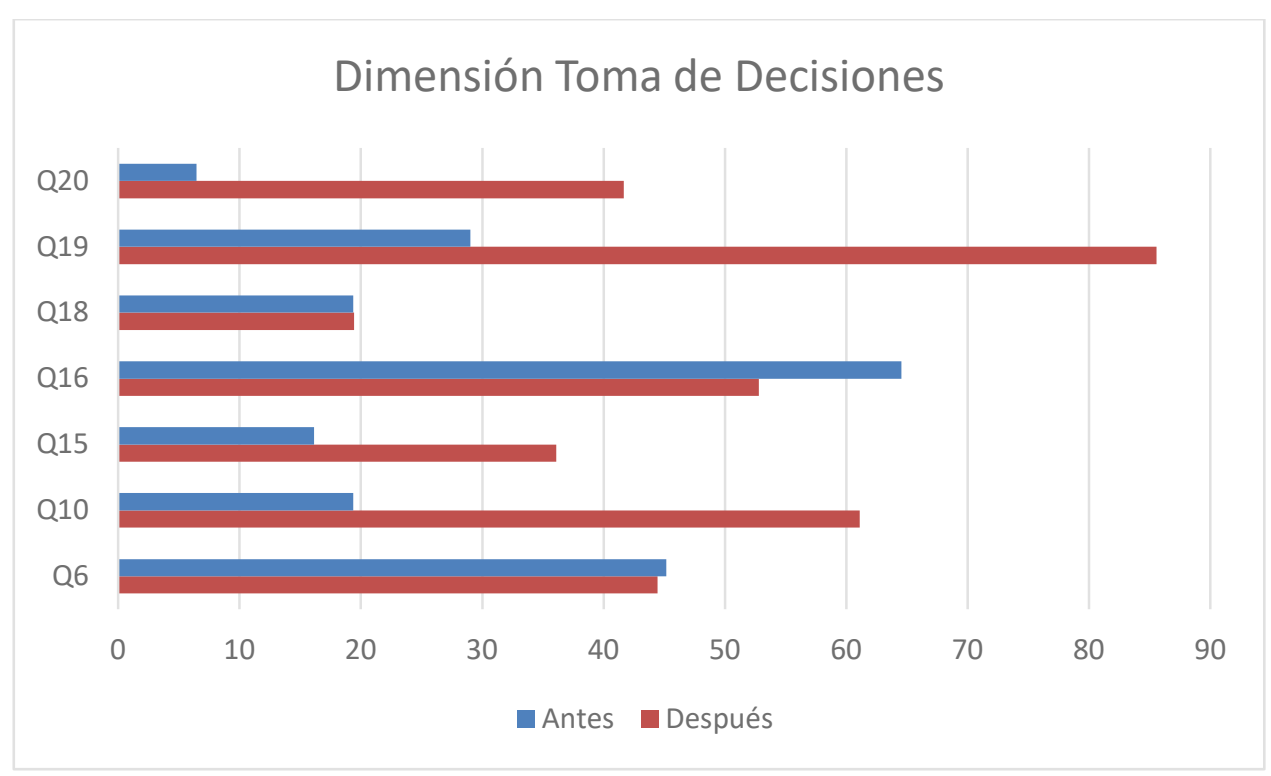

Fig. 8. Porcentajes aciertos a las preguntas dentro de la dimensión crítica

\section{Conclusiones}

En general los resultados muestran que el porcentaje de respuestas correctas han aumentado, y en algunos casos de forma significativa con p-valores pequeños. En especial en las dimensiones de cálculo y pensamiento crítico, lo que enfatiza el buen funcionamiento del aprendizaje basado en proyectos en dichas áreas. La actividad propuesta y el análisis de los resultados permite la evaluación de las competencias transversales de la asignatura.

En cuanto a la sinergia entre el aprendizaje basado en proyectos y el ODS 7, se ha consiguido que los alumnos trabajen con un problema real relacionado directamente con la optimización del recurso eólico y 
el análisis de viabilidad del proyecto, conceptos que están totalmente integrados en los proyectos propuestos dentro de este objetivo.

No obstante, cabe destacar que los resultados obtendiso son de un solo año no y no son suficientes para establecer las conclusiones de forma global. En este sentido, sería necesario ver la evolución en años posteriores y la comparación de las mismas dimensiones con otros aspectos que no se trabajan con el aprendizaje basado en proyectos.

\section{Agradecimientos}

Este trabajo se ha realizado en el marco del Proyecto de Innovación y Mejora Educativa, PIME Curso 2018-2019 "Coordinación de competencias transversales en asignaturas de ámbito nuclear en el Grado de Ingeniero de la Energía", Referencia B16, del Vicerrectorado de Estudios, Calidad y Acreditación de la Universitat Politècnica de València.

\section{Referencias}

CUIÑAS I., MARIÑO-ESPIÑEIRA P., FERNANDEZ-IGLESIAS M., CAEIRO, M., COSTA-MONTENEGRO E., DIAZ-OTERO F. (2016). Evaluación de competencias con metodologías de aprendizaje basado en proyectos. Congreso INRED 2016

GARCIA, J., PEREZ, J. (2018) Aprendizaje basado en proyectos: método para el diseño de Actividades. Revista Tecnología, Ciencia e Innovación, CEF, núm. 10 (mayo-agosto 2018, pp. 37-63

HUFF, J. L., ZOLTOWSKI, C. B. AND OAKES, W. C. (2016), Preparing Engineers for the Workplace through Service Learning: Perceptions of EPICS Alumni. Journal of Engineering Education. 105,43-69.

MARTON I., GALLARDO S., VILLANUEVA J.F., CARLOS S., SÁNCHEZ A., (2019) Aprendizaje basado en proyectos en el Grado en ingeniería de la energía, Congreso INRED 2019.

NACIONES UNIDAS. (2019) Objetivos y metas de desarrollo sostenible.

$<$ https://www.un.org/sustainabledevelopment/es/objetivos-de-desarrollo-sostenible/ >[consulta: Marzo 2019] . 